\title{
Las competencias ciudadanas en la clase de Educación Física: entre las miradas desde la docencia y el discurso planteado en el currículo dominicano
}

Prada Rozo, Mauricio Javier

Las competencias ciudadanas en la clase de Educación Física: entre las miradas desde la docencia y el discurso planteado en el currículo dominicano

Revista Educación, vol. 45, núm. 1, 2021

Universidad de Costa Rica, Costa Rica

Disponible en: http://www.redalyc.org/articulo.oa?id=44064134025

DOI: https://doi.org/10.15517/revedu.v45i1.41402

Esta obra está bajo una Licencia Creative Commons Atribución-NoComercial-SinDerivar 3.0 Internacional. 


\title{
Las competencias ciudadanas en la clase de Educación Física: entre las miradas desde la docencia y el discurso planteado en el currículo dominicano
}

\author{
Citizenship Skills in Physical Education Class: Teaching and the Discourse Proposed for the Dominican \\ Curriculum
}

Mauricio Javier Prada Rozo

Instituto Superior de Formación Docente Salomé Ureña, República Dominicana

mauricio.prada@isfodosu.edu.do

(iD http://orcid.org/0000-0001-9752-0552

\author{
DOI: https://doi.org/10.15517/revedu.v45i1.41402 \\ Redalyc: http://www.redalyc.org/articulo.oa?id=44064134025
}

Recepción: 01 Mayo 2020

Aprobación: 20 Septiembre 2020

\section{Resumen:}

La formación en ciudadanía se ha convertido en una de las principales estrategias para disminuir los problemas sociales, tales como la violencia, el cuidado del medio ambiente y la participación democrática. Esta responsabilidad involucra a la escuela y su contexto como un espacio de interacción social. Con este trabajo se pretende identificar el nivel de apropiación e implementación de las competencias ciudadanas o fundamentales propuestas en el currículo de la República Dominicana y establecer una relación entre lo que manifiesta el Diseño Curricular - Primer Ciclo de Secundaria (DC-PS) y lo planteado en la clase de Educación Física. Este estudio es de tipo cualitativo, con datos suministrados por docentes, tanto de forma individual como en colectivo, por medio de entrevistas semiestructuradas y grupos de discusión. El análisis se efectuó a través del programa ATLAS.ti. Se diseñó un esquema con las categorías emergentes: grado de dominio y actividades implementadas, y la participación de la comunidad que interactúa con del estudiantado. El proyecto se desarrolló en el 2019, en la ciudad de Santo Domingo, en una N=55 docentes, por medio de un muestreo intencional no probabilístico. Con este estudio, se pudo concluir que el profesorado de Educación Física contribuyen a la formación en Competencias Fundamentales a través del desarrollo de las actividades lúdico deportivas; pero estas se plantean como un valor agregado de la actividad desarrollada y de forma no planificada; sin embargo, ante las dificultades de tipo comportamental, el cuerpo docente está presto a solucionarlas de la mejor manera, tomando como base su propia formación en valores sociales y culturales y no como lo manifiesta el DC-PS. Se recomienda crear estrategias pedagógicas que permitan al profesorado organizar actividades específicas para el fortalecimiento de las Competencias Fundamentales, que permita la unificación de los criterios entre docentes, y así ser implementadas en las instituciones educativas, al involucrar a la familia y la comunidad.

Palabras Clave: Educación secundaria, Competencias Fundamentales, Entrevista, Educación Física, Docencia.

\section{Abstract:}

Teaching citizenship skills has become an important strategy for reducing social problems, such as violence and fostering positive responses, such as environmental protection and democratic participation, a responsibility that is taught within the school setting as a place for constant social interaction. This study attempts to identify the level of appropriation and implementation of citizenship and essential skills in the school curriculum of the Dominican Republic, thus, establishing a relationship between the Curricular Design for the First Cycle of High School (DC-PS) and what is proposed for Physical Education class. This is a qualitative study with data provided, individually and collectively, by teachers based on semi-structured interviews and discussion groups. The analysis was done through the ATLAS.ti program. An outline was designed which included the following emerging categories: degree of mastery of the implemented activities and student engagement with the community. The project was developed in 2019, in the city of Santo Domingo with an intentional non probabilistic sample of $\mathrm{N}=55$ teachers. The study concluded that Physical Education teachers contribute to developing essential skills through sports and recreational activities as an added value executed in an unplanned manner. Yet, in the face of students experiencing behavioral difficulties, teachers are ready to resolve any issues in the best manner possible using their social and cultural training in contrast to what is stated in the DC-PS. A recommendation is for teaches to create teaching strategies to better organize activities for strengthening essential skills so that teaching criteria may be standardized and, thus implemented at educational institutions as well as involve the family and community.

KEYWORDS: High School Education, Fundamental Skills, Interview, Physical Education, Teaching. 


\section{INTRODUCCIÓN}

En los últimos años se ha buscado implementar una educación integral, donde el estudiantado sea el actor principal, al involucrar en su formación, el fortalecimiento socioemocional y el desarrollo de competencias (Levoratti, 2014). Es aquí donde las prácticas pedagógicas cobran vital importancia, pues el profesorado asume la responsabilidad de fortalecer en los educandos, las diversas competencias educativas como lo son las ciudadanas (Zambrano, 2018).

El Ministerio Nacional de Educación de la República Dominicana (MINERD), plantea en el currículo educativo, la formación cívica ciudadana, por medio de las denominadas Competencias Fundamentales (CF), las cuales se proponen ser desarrolladas de forma trasversal durante los años de formación de la población estudiantil.

Por lo anterior, es importante que el personal docente, en su condición de líder y como agente activo en la formación del estudiantado, asuma la responsabilidad de generar espacios donde se ponen a prueba las CF, al igual que plantear objetivos de enseñanza específicos dentro de las clases, con el fin del fortalecerlas.

Esta responsabilidad no solo es de las y los docentes y la escuela, sino que existen otros factores sociales de gran impacto en el fortalecimiento de las CF, como lo son: la globalización, el consumismo, la conformación de la familia y las tecnologías de la Información y la Comunicación (TIC), entre ellas, las redes sociales (Maldonado, 2018).

Este trabajo pretende identificar el nivel de apropiación e implementación de las CF durante la clase de Educación Física, tratando de establecer una relación entre los que manifiesta de manera explícita el Diseño Curricular de Primer Ciclo de Secundaria (DC-PS) y el nivel de apropiación del profesorado al igual que las estrategias implementadas desde la escuela.

Se toma como categorías emergentes el grado de dominio y actividades implementadas por el grupo docente, pues estas permiten identificar si existe una apropiación desde el aspecto cognitivo sobre las CF y si estas se llevan a la práctica durante el desarrollo de las clases. Una segunda categoría es la participación de la comunidad que interactúa con el alumnado, ya que como lo manifiesta Zambrano (2018), la formación en ciudadanía debe ser constante y se debe manejar la misma información en los distintos contextos donde se desenvuelven jóvenes y adolescentes; esta categoría analiza la participación de la comunidad que interactúa con estudiantes y el apoyo administrativo e institucional.

\section{Antecedentes}

El fortalecimiento de las competencias es esencial en la formación de ciudadanía, tal y como lo propone Monzonís y Capllonch, (2015), quienes desarrollaron un trabajo bajo la metodología investigación acción, implementada en un centro de educación primaria en Barcelona, España. El objetivo del trabajo era, "la adquisición de las capacidades de la competencia social y ciudadana del alumnado con la intención de prevenir y resolver positivamente los conflictos y, mejorar con ello el clima de convivencia en el aula" (Monzonís y Capllonch, 2015, p. 256), favoreciendo con ello la convivencia en el contexto escolar desde el área de Educación Física por medio de tutorías.

Dentro de los resultados obtenidos, lograron sensibilizar al estudiantado frente a la forma adecuada de resolver conflictos. Esta experiencia permite establecer que la orientación y seguimiento consensuado a los problemas que presenta el alumnado en el aula de clase, puedan servir como medio para el fortalecimiento de habilidades sociales.

En esta misma línea, se hace un acercamiento a la propuesta de Levoratti (2014), quien realiza una consulta a las últimas reformas educativas implementadas a partir del año 2006 en Buenos Aires, Argentina. Revisando la Educación Física como asignatura escolar, y quienes pretenden “....analizar cuáles son los fundamentos 
y las concepciones circulares en torno al juego y al deporte como práctica que contribuye a la formación ciudadana" (p. 1), por ello, hace una revisión minuciosa de las metodologías propuestas en los currículos y cómo a partir de un enfoque adecuado con objetivos claros, se podría propiciar el aprendizaje de prácticas democráticas, logrando la "inclusión [, el aprendizaje de determinados valores y promoviendo] hábitos de salud [adecuados]” (Levoratti, 2014, p. 7).

Dentro de los hallazgos significativos, se encuentra la categorización de lo que se debe entender por deporte escolar, pues existe una disonancia entre los objetivos curriculares de la asignatura y los imaginarios del grupo docente que la imparte, quienes canalizan el trabajo en el aula más hacia el deporte competitivo y no al deporte escolar, el cual busca la formación del individuo desde todas sus dimensiones (Levoratti, 2014). Por lo anterior es importante identificar los imaginarios sociales que se presentan en el profesorado y directivos, en relación con la práctica de la Educación Física y de qué manera afecta la formación ciudadana de los educandos.

Otras investigaciones han logrado demostrar que las actividades lúdicas recreativas son una estrategia pedagógica de gran impacto en la formación de la ciudadanía, ya que este tipo de actividades son más llamativas entre el estudiantado, requieren de una alta capacidad de autoconocimiento socioemocional, al momento de interactuar con los compañeros y al afrontar los problemas de forma asertiva, controlando las emociones propias en los momentos de tensión y una constante comunicación durante las actividades planteadas, reacciones que requieren de una alta resiliencia y empatía (Monzonís y Capllonch, 2015; Prada, 2018).

Por lo anterior se puede evidenciar el esfuerzo ejecutado en la formación para la ciudadanía, ya que la falta de esta se ve reflejada en problemas sociales (Alarcón y Donoso, 2018), los cuales desencadenan en violencia, consumo de sustancias psicoactivas, maltrato al medio ambiente, entre otros.

La República Dominicana no es ajena a esta situación; estudios desarrollados por el Sistema Regional de Evaluación y Desarrollo de Competencias Ciudadanas, en el marco del Estudio Internacional de Educación Cívica y Ciudadanía - ICCS, por sus siglas en inglés, dejó en evidencia una pobre formación en competencias cívicas del estudiantado dominicano. El mismo estudio resalta que la principal problemática se encuentra en las escuelas del sector público, en las comunidades con menor acceso a recursos educativos y padres con bajo nivel de formación académica (Schulz, Ainley, Fraillon, Kerr y Losito, 2010). Estos resultados invitan a evaluar y reflexionar sobre las estrategias implementadas para la formación en ciudadanía y si involucran a las poblaciones vulnerables, a las familias, tutores y a la comunidad donde desenvuelven los jóvenes y adolescentes diariamente.

La anterior información es corroborada por otro estudio elaborado en América Latina sobre la violencia en la escuela, el cual manifiesta que la República Dominicana presenta un alto grado de robos, amenazas y ridiculizaciones por parte del estudiantado a los compañeros de escuela. Esta también dejó en evidencia una tendencia por parte del grupo docente y directivos al reforzar, de manera inconsciente, la discriminación de género en actividades cotidianas, asignando labores de aseo o limpieza a las niñas y actividades de fuerza a las niñas y los niños, reforzando estigmas sociales y la división sexual del trabajo (Pacheco, 2017), lo que va en contra del objetivo de la formación ciudadana, la cual intenta dotar a estudiantes de herramientas que le permitan valorar el entorno, por medio de un análisis crítico de la realidad (Ospina, 2016), con la capacidad de intervenir y transformar positivamente el contexto donde se desenvuelve.

\section{Marco teórico}

Al incorporar las competencias ciudadanas como parte de las estrategias pedagógicas, se fortalece el desarrollo de habilidades para la toma de decisiones, la adquisición de criterios, el trabajo en equipo, la administración eficiente del tiempo, la asunción de responsabilidad, la solución de conflictos y problemas, al igual que las habilidades para la comunicación, la negociación y la participación democrática (MINERD, 2016), por lo anterior se plantean en el currículo dominicano las Competencias Fundamentales, entendiendo estas como 
el principal mecanismo para asegurar la coherencia del proyecto educativo. Por su carácter eminentemente transversal... esenciales para el desarrollo pleno e integral del ser humano en sus distintas dimensiones, se sustentan en los principios de los Derechos Humanos y en los valores universales... y participación en los procesos democráticos (MINERD, 2016, pp. 38-39).

Planteando para su desarrollo las siguientes competencias fundamentales: "Ética y Ciudadana [,] Comunicativa, del Pensamiento Lógico, Creativo y Crítico [,] Resolución de Problemas [,] Científica y Tecnológica [,] Ambiental y de la Salud [y] Desarrollo Personal y Espiritual” (MINERD, 2016, p. 67).

Para este estudio se adopta la definición de competencia, planteada por el MINERD (2016) como

La capacidad para actuar de manera eficaz y autónoma en diversos contextos, movilizando de forma integrada conceptos, procedimientos, actitudes y valores...Las competencias se desarrollan de forma gradual en un proceso que se mantiene a lo largo de toda la vida; tienen como finalidad la realización personal, el mejoramiento de la calidad de vida y el desarrollo de la sociedad en equilibrio con el medio ambiente. (MINERD, 2016, p. 38).

Y se complementa con lo propuesto por Prada (2018), quien manifiesta que la competencia es la destreza adquirida por una persona de ejecutar una actividad con causa de conocimiento, desde el saber, el hacer y el saber hacer.

Por otro lado, se entiende la Educación Física no solo como el cuerpo humano y su capacidad de movimiento, sino más bien como el sujeto, que por medio del movimiento, se forma como un ser social y político, con capacidad de comunicación tanto verbal como corporal, capaz de generar relaciones sociales y culturales (Cagigal, 1982), convirtiéndola en una herramienta importante en la formación de ciudadanía.

El concepto de competencias ciudadanas o fundamentales se vuelve controversial para la formación, esto debido a que contienen componentes biológicos, sociales, políticos y culturales que influyen en los procesos de aprendizajes de los individuos.

Es así que desde los componentes biológicos, la práctica de la actividad física estimula áreas del cerebro encargados del control emocional, al igual que la toma de decisiones y las respuestas automáticas ante las situaciones de conflicto, como lo es la corteza pre frontal, al igual que la memoria de trabajo, el control inhibitorio y la flexibilidad mental (Baggetta y Alexander, 2016), los cuales son necesarios para el razonamiento, la resolución de problemas y la planificación (Pherez, Vargas y Jerez, 2018).

Desde los componentes sociales, las Competencias Fundamentales son influenciadas a lo largo de la vida, pues son parte de la formación de principios y valores sociales, y morales, esenciales en el desarrollo del ser. Una adecuada interacción con las demás personas, con la naturaleza y en diferentes contextos, implica el fortalecimiento de habilidades sociales, control de las emociones y valores que le permitirán desenvolverse con éxito dentro de la sociedad (Zambrano, 2018). Por eso, en el fortalecimiento de estas competencias se debe abordar al individuo desde varias alternativas pedagógicas, vivenciales y no solo desde la parte formativa magistral.

\section{Metodología}

El presente estudio es de tipo cualitativo, con datos suministrados por docentes de forma individual y en colectivo; el proyecto se desarrolló entre los meses de enero y diciembre de 2019, en el distrito 15-05 de la ciudad de Santo Domingo, República Dominicana, donde se seleccionaron 63 docentes de Educación Física por medio de un muestreo intencional no probabilístico (Otzen y Manterola, 2017).

La recolección de la información se efectuó en las instalaciones de la universidad, donde 47 docentes participaron en grupos de discusión y ocho en entrevistas individuales.

Los criterios de selección fueron: docentes licenciados/as en Educación Física, debidamente contratados por la institución educativa del distrito seleccionado, con más de cinco años activos en esta área y que tuvieran a su cargo estudiantes de primer ciclo de nivel secundario. 
Los grupos de discusión se organizaron en cuatro sesiones, cada una con un promedio de 12 participantes y la última sesión con 11; cada grupo representativo del contexto y conformado por docentes de ambos géneros.

Se implementaron ocho entrevistas en profundidad a docentes de Educación Física del mismo distrito, estas fueron implementadas por una psicóloga, quien es parte del grupo de investigación y cuenta con amplio conocimiento en el tema.

Una vez expuestos los compromisos por parte del grupo de investigación, sobre la forma de salvaguardar la confidencialidad de la información, según la declaración de Helsinki, los participantes diligenciaron los consentimientos informados y dieron autorización para permitir la grabación en voz (Asociación Médica Mundial [AMM], 2019).

La recolección de la información se organizó por medio de la entrevista semiestructurada y de grupos de discusión.

Las preguntas se diseñaron con base en lo expuesto en el currículo dominicano, en especial con la "Evaluación de los aprendizajes" (MINERD 2016, p. 56), el cual indica que el profesorado debe poseer las competencias suficientes tanto para la enseñanza como para la evaluación de las competencias fundamentales; también se tomaron en cuenta los "Niveles de dominio" (MINERD, 2016, p. 67) por competencia y se siguieron los lineamientos planteados por Folgueiras y Ramírez (2017), elaborando un guion de preguntas, las cuales fueron revisadas por expertos en el tema.

Una vez aprobadas por el grupo de expertos, las entrevistas fueron aplicadas a cinco docentes de Educación Física, con el ánimo de identificar si las preguntas eran abiertas, si permitían recolectar la información necesaria y si las respuestas brindaban la posibilidad de efectuar nuevas preguntas (Bohórquez y Checa, 2017).

Las grabaciones obtenidas de las entrevistas y de los grupos de discusión fueron transcritas por el equipo de investigación, para luego ser leídas y releídas con el fin de clasificar e interpretar la información recolectada y así crear un esquema de análisis (Marín, Hernández y Flores, 2016); el cual fue registrado en el programa ATLAS.ti versión 7.1.3, tomando como base las categorías emergentes de las preguntas ejecutadas en la entrevista y el objetivo de la investigación (Blanco, 2012).

Se identificaron palabras, frases y segmentos de las conversaciones, se resaltó la información relevante y se la ordenó de forma tal que permitiera encontrar cosas en común, diferencias, patrones y estructuras. De este análisis, surgieron cuatro categorías, las cuales fueron agrupadas en dos ejes, como se puede observar en la Tabla 1.

\section{TABLA 1}

Categorización de las entrevistas y grupos de discusión

\begin{tabular}{l|l} 
Eje 1 & Grado de dominio y actividades implementadas \\
\hline Categoría 1 & Aspectos cognitivos sobre las Competencias Fundamentales. \\
\hline Categoría 2 & Estrategias para su fortalecimiento en el estudiantado. \\
\hline Eje 2 & Participación de la comunidad que interactúa con estudiantes \\
\hline Categoria 1 & Participación de la comunidad que interactúa con estudiantes \\
\hline Categoría 2 & Apoyo administrativo institucional y actividades con la población del entorno.
\end{tabular}

Fuente: Elaboración propia.

Fuente: Elaboración propia.

Para el análisis de los resultados, se presentan en este artículo solo las declaraciones más representativas.

La información obtenida durante el grupo de discusión se encuentra codificadas con la letra $G$ y la información de la entrevista con la letra $\mathrm{E}$, continuando con la clasificación de género, si es profesor o profesora y la edad de la persona entrevistada. Esta información permite una mayor confianza al análisis, al igual que facilita la triangulación de la información (Palomares, Dharmadi, Sulistia, Collado y Padial, 2018). 
La contrastación se efectuó por semejanza y diferencias entre las categorías, soportando la información que surge y contrastándola con los marcos teóricos de referencia, con el fin de obtener una descripción de la realidad del problema (Delgado y Castrillo, 2015).

La teorización se llevó a cabo de acuerdo con lo indicado por la bibliografía, elaborando nuevas hipótesis, esquematizaciones y modelos, con el fin de dar explicación a los hallazgos que emergen del estudio e interpretando los resultados obtenidos (Guerrero, 2016).

\section{ANÁLISIS Y DISCUSIÓN DE RESULTADOS}

En el eje: Grado de dominio y actividades implementadas, los resultados en la categoría Aspectos cognitivos sobre las Competencias Fundamentales, se evidencia una marcada tendencia a confundir las competencias específicas, planteadas para el curso, con las Competencias Fundamentales.

Profesor, G, 49 años: "estas [las Competencias Fundamentales] se desarrollan en la clase, se realizan ejercicios de trote para mejorar la resistencia, sal tos para la fuerza y la coordinación" (Comunicación personal, 2019).

A pesar de tener un conocimiento generalizado sobre la importancia del fortalecimiento de las Competencias Fundamentales en la formación de los jóvenes y adolescentes, en algunos casos se deja entrever que el concepto de Competencias Fundamentales varía notablemente con lo planteado en el currículo dominicano, y en otros casos, se entiende el término de competencias como sinónimo de competitividad.

Profesor, E, 52 años:

Yo siempre les digo a mis estudiantes que tienen que ser los mejores, que tienen que ganar en todo, que la frase lo importante es competir' fue hecha por perdedores, uno siempre debe buscar ganar como sea, el primero es primero y lo importante es ganar, ganar es lo único importante en la vida (Comunicación personal, 2019).

Profesor, G, 39 años: "Bueno, las competencias se trabajan en la clase desde actividades de juegos deportivos, conformando grupos y compitiendo entre ellos" (Comunicación personal, 2019).

Profesora, E, 47 años:

Son [las Competencias Fundamentales] las estrategias básicas sobre las cuales se va a desarrollar el trabajo y son los elementos, con base en los cuales, vamos a formar a nuestros estudiantes, que sean competentes, en los tiempos que estamos viviendo (Comunicación personal, 2019).

Profesor, G, 51 años: "Son [las Competencias Fundamentales] los componentes en lo cualitativo y en lo cuantitativo, para un desenvolvimiento del desarrollo, que sea ecuánime, que se acorde con las necesidades" (Comunicación personal, 2019).

Profesora, E, 40 años: "Según nuestro currículo dominicano, las Competencias Fundamentales son las competencias esenciales para el desarrollo del ser humano, que nos permitan, hummm, desarrollarlo en distintas dimensiones, tomando en cuenta los valores fundamentales y los derechos humanos" (Comunicación personal, 2019).

En la categoría Estrategias para su fortalecimiento en el estudiantado, se pretende identificar si las actividades desarrolladas por el grupo docente en la clase de Educación Física tienen como objetivo fortalecer las Competencias Fundamentales y qué actividades se ejecutan en la clase para este fin.

Profesor, G, 47 años:

Bueno, yo siempre trato de hablarles a los estudiantes sobre lo que pasa en la sociedad, me entiende, les hablo sobre los peligros de la calle, que deben cuidarse y dejar los malos amigos, me entiende, trato de darles consejos (Comunicación personal, 2019).

Profesor, G, 39 años: 
Bueno, actividades específicas para desarrollar las Competencias Fundamentales, no, pero dentro de las actividades que se hacen en una clase, en las actividades deportivas o de juegos, va inmerso el desarrollo de las competencias, uno se da cuenta cuando los estudiantes alegan [discuten] y entonces uno los corrige (Comunicación personal, 2019).

Profesor, E, 49 años:

Debemos tener en cuenta, específicamente, que lo psicológico juega un papel fundamental en cada uno de esos tópicos [Se le pide al/la docente que explique, ¿Por qué desde lo psicológico?] ... Claro, porque con los estudiantes, tú tienes que forjarlos con elementos positivos, que tú sepas que deben tener una autoestima bien elevada y decir, yo puedo, yo puedo, yo puedo (Comunicación personal, 2019).

En la información suministrada por el profesorado, se deja entrever que las Competencias Fundamentales no están establecidas como un objetivo por desarrollar dentro de la clase, ni se encuentra estructurada en la planificación diaria para dicho fin.

Se asume por el personal docente que al organizar actividades grupales, juegos colaborativos o competitivos, ya se está formando en Competencias Fundamentales; si bien esta suposición es cierta, pues una de las ventajas que tiene la Educación Física es que requiere del desarrollo de estas competencias, las cuales serían más efectivas si se plantean como objetivos de las clases, acompañadas de una retroalimentación y reflexión, tomando como ejemplo los problemas del contexto donde viven los jóvenes y adolescentes.

Profesora, E, 45 años: "Se organizan torneos, yo hago gimnasiadas; en las gimnasiadas yo logro todas esas Competencias Fundamentales que estoy buscando" (Comunicación personal, 2019).

Profesor, G, 49 años: "La Educación Física es una de las áreas donde más se puede desarrollar las Competencias Fundamentales, ya que este [las y los estudiantes], socializa, resuelve problemas, puede crear y así sucesivamente" (Comunicación personal, 2019).

Al tratar de indagar sobre la forma en la cual el profesorado determina el nivel de apropiación de las Competencias Fundamentales en estudiantes, este manifiesta que se desarrolla por medio de los indicadores de logro planteado en el currículo dominicano, el cual consiste en efectuar una evaluación actitudinal al inicio del año escolar; sin embargo, estas pruebas son específicas para determinar las competencias específicas. Durante esta evaluación, el personal docente, de forma subjetiva, mira o evalúa el nivel de apropiación de las Competencias Fundamentales que presenta el estudiantado al momento de ingresar a la institución.

Profesor, G, 47 años:

$\mathrm{Al}$ ingreso del año se realizan unas pruebas, donde aparte de las pruebas de aptitud física, también nos damos cuenta de los valores de los diferentes puntos de las Competencias Fundamentales, con las que ellos [los estudiantes] vienen y las que aún les faltan por alcanzar (Comunicación personal, 2019).

Profesora, E, 49 años:

Las Competencias Fundamentales es lo que se espera que el niño quiera lograr, ya al final del año escolar, ya al final se quiere que el domine esas siete Competencias Fundamentales, donde el este apto para integrarse a la sociedad, de forma productiva... se realiza una diagnostica, donde se dialoga con el niño sobre los conocimiento que él tiene sobre el tema y entonces, dependiendo los niveles, se realizan accione en pro del desarrollo de esas competencias (Comunicación personal, 2019).

En el eje: Participación de la comunidad que interactúa con estudiantes, relacionado con la categoría Apoyo administrativo institucional y actividades con la población del entorno, se pretende indagar sobre el papel que está desempeñando el área administrativa en relación con las Competencias Fundamentales, tales como espacios, recursos y apoyo para el desarrollo de actividades que pretendan fortalecer dichas competencias en el estudiantado de los planteles educativos, involucrando a la comunidad del entorno (padres de familia, vecinos, tutores y demás personas que se encuentran en contacto con estudiantes), pues influyen de forma directa e indirecta en su formación.

Según la gestión administrativa, el personal docente manifiesta que este se centra más hacia las actividades deportivas competitivas, que a la formación en Competencias Fundamentales, ya que encuentra mayor apoyo 
cuando las actividades le permiten a la institución educativa darse a conocer y sobresalir ante las demás instituciones por su rendimiento deportivo; sin embargo, la mayoría de docentes manifestó que a pesar de existir los recursos para la educación, estos no llegan a tiempo a las instituciones educativas o simplemente no llegan, lo que les deja sin herramientas para ejecutar el trabajo formativo. Lo cual se contradice con lo planteado por el MINERD (2016), en el currículo dominicano, pues este establece en su apartado "Los medios y recursos para el aprendizaje” (p. 52), que la clase de Educación Física debe contar con la dotación de "equipos deportivos y recreativos" (p. 56).

Profesora, G, 45 años:

Bueno, viene la convivencia que hacemos con mucho o poco... son competencias que hacen que los niños se compenetren con otras escuelas. ... O sea se hacen arañando, [con pocos recursos], pero imagínese esa es la realidad de nosotros (Comunicación personal, 2019).

Profesor, E 48 años: "Independientemente yo hago mi programación y el centro me brinda soporte de una forma u otra, ya sea moral o ya sea económico" (Comunicación personal, 2019).

Profesor, G, 45 años:

Las instituciones organizan los juegos deportivos en los cuales participan las escuelas en todos los deportes, sin embargo y a pesar de estar estipulado en el POA [Plan Operativo Anual], los recursos económicos no llegan a la institución y a veces toca poner del bolsillo de uno, pero no siempre se puede (Comunicación personal, 2019).

Profesor, G, 47 años:

Las entidades organizan los juegos deportivos, pero para preparar a los estudiantes en estos juegos, las escuelas no cuentan con material; en mi escuela tenemos un solo balón de baloncesto para 600 niños y así no se puede (Comunicación personal, 2019).

En la categoría Actividades con la población del entorno, se tomó en cuenta que la formación en ciudadanía no solo es responsabilidad del profesorado y de la escuela, se deben involucrar las personas y entidades que tiene relación directa e indirecta con la formación de jóvenes y adolescentes, es por esto que la formación en Competencias Fundamentales se debe abordar desde varios estamentos, como son los medios de comunicación, las tecnologías, las organizaciones sociales, las familias, entidades gubernamentales, entre otros.

Otras de las problemáticas sociales que se presentan en este eje están relacionadas con cambios sociales, actuales, como el aumento de madres cabeza de hogar y los cambios en la conformación de las familias, haciendo que la participación de padres y tutores en las actividades planteadas por la escuela y el profesorado, sea casi nulo.

Profesora, G, 48 años: "Lamentablemente estamos careciendo de actividades con la comunidad" (Comunicación personal, 2019).

Profesor, E, 52 años:

Es que la comunidad ha cambiado mucho y la comunidad ahora es un poco dejada y no quiere dejar su casa, ya no le preocupan tanto los hijos, cuando usted los llama, para llamar la atención por una eventualidad que su hijo cometió en un día, indisciplina por ejemplo y si el padre no tiene una cuota de responsabilidad de pagar el daño que hizo, el padre lo deja pasar fácilmente (Comunicación personal, 2019).

Profesor, G, 43 años: "Allá en mi centro yo realicé un intramuros, invité a dos clubes deportivos para participar, invité algunos maestros e invité también a padres de la comunidad, pero los padres de la comunidad no fueron" (Comunicación personal, 2019).

En otros casos se deja entrever la importancia de la relación del grupo docente con las distintas entidades y agrupaciones de líderes comunitarios para el apoyo de las actividades planteadas.

Profesor, G, 52 años: 
Si los docentes que no trabajan en el sector donde viven se dificulta más, ya que no conocen la junta de vecinos, no conocen los dueños de clubes y eso, en mi caso sí recibo apoyo de la comunidad ya que los conozco porque yo vivo en la zona (Comunicación personal, 2019).

Cuando se analizan las respuestas dadas por el profesorado, se deja entrever, que la participación de la comunidad en las actividades planteadas es muy baja, en algunas ocasiones por el mismo desinterés de los padres de familia por participar en las actividades de la escuela, y en otros casos por la falta de un programa estructurado por parte de la institución educativa que permita crear conciencia en la comunidad y en los padres de familia sobre la importancia de estos en la formación de sus estudiantes.

Se observa en la información suministrada por el personal docente, resultados similares a los presentados por Cárcamo (2015), donde se evidencia la existencia de una representación idealizada de la ciudadanía, reforzada por la formación de docentes y la influencia de la formación cultural, que busca preservar patrones de conducta dentro de las clases como la disciplina, la autoridad docente y el respeto; las cuales le facilitan el desarrollo de los objetivos planteados para la clase. La tendencia en las respuestas del profesorado se inclina a la formación cívica, viendo esta como el ciudadano respetuoso de las leyes y de las normas, en busca del bien de la sociedad, sin embargo predomina en el grupo docente un imaginario del respeto, la autoridad y el acatamiento de estas normas de forma autoritaria, lo que desvirtúa el objetivo de la formación ciudadana, haciendo énfasis solo en la formación de valores e intereses particulares, emanados de las ideologías del profesorado y de la creencia acerca del ciudadano de bien.

En la categoría Estrategias para el fortalecimiento de las Competencias Fundamentales en estudiantes, existe la tendencia a confundir la competencia planteada desde la capacidad del saber hacer, con la competencia deportiva; reforzado por el imaginario social de ver al grupo docente de Educación Física como el profesor de deportes (López, Pérez, Manrique y Monjas, 2016), por lo tanto, las actividades planteadas por el profesorado durante la sesión de clase se orienta más a las actividades deportivas competitivas. Esta mirada deportivista, se ve reforzada por las entidades que regulan la Educación Física en la República Dominicana y afecta de forma indirecta los objetivos dirigidos a la formación cívica ciudadana, ya que la competitividad no se asume como parte de una rivalidad respetuosa, sino que predomina la tendencia a ganar a toda costa, sin respetar las normas o buscando la forma de hacer trampa para sacar ventaja sobre el oponente (Agüero, 2015).

Si bien, el currículo dominicano plantea dentro de los niveles de dominio los componentes que se requieren para desarrollar las Competencias Fundamentales, según la información suministrada por las y los docentes, no se guarda una relación directa entre el currículo dominicano y lo planteado en una clase de Educación Física, lo que deja entrever la influencia de un currículo oculto o también llamado tácito, latente o no escrito, entendiendo este como las actividades que ejecuta el profesorado "a través de los modos de ver y de comunicar, y también, mediante la adquisición de costumbres y rituales asumidos como normales e incluso, deseables" (Herrera, 2016, p. 2), influyendo en docentes y en estudiantes tanto en el currículo formal como en el no formal (Mossop, Dennick, Hammond y Robbé, 2013).

Otro indicador importante para determinar el grado de avance del estudiantado y el cumplimiento de los objetivos planteados para el desarrollo de las clases, es la evaluación, dentro de esta el MINERD plantea los criterios de evaluación, los componentes y elementos que orientan al profesorado sobre el tipo de aprendizaje que debe alcanzar cada estudiante por Competencia Fundamental, señalando los aspectos sobre los cuales el conjunto docente determina el nivel de apropiación de la competencia en el alumnado (MINERD, 2016) y es por medio de los indicadores de logro. Sin embargo, estos indicadores están diseñados para determinar el fortalecimiento de las competencias específicas y no para las Competencias Fundamentales (MINERD, 2016); por lo tanto, se debe fortalecer en el profesorado la forma adecuada de evaluar e identificar de una forma objetiva, el nivel de desarrollo de las Competencias Fundamentales.

Continuando con el eje Participación de la comunidad que interactúa con estudiantes, en lo relacionado con la categoría Apoyo administrativo, se sigue evidenciando en las respuestas de la entrevista y grupos de discusión, una fuerte tendencia hacia las actividades deportivas, donde los objetivos están más enfocados a 
la representatividad de la institución en los resultados y el rendimiento presentado en los juegos deportivos escolares, actividades que si no son bien encaminadas pueden generar una formación negativa en la educación ciudadana, al volverse discriminativa y displicente, al plantearse como objetivo principal ganar los eventos a toda costa, con el fin de adquirir los beneficios propuestos (Lamoneda y Huertas, 2017). Esta forma de actuar estaría en contra de lo que buscan las Competencias Fundamentales.

Unas de las características que surgen del proceso de investigación son las actividades con la población del entorno, a pesar de la importancia que tiene el involucrar a la comunidad, padres de familia y tutores, en la formación para la ciudadanía de jóvenes y adolescentes, esta no se incluye dentro de las estrategias implementadas para la formación en ciudadanía, como la plantea Martínez e Ibarrola (2015), si una persona cuenta con un núcleo familiar, sin importar el tipo de conformación, este tiene mayor posibilidades de integrarse mejor en una sociedad, impulsando a que estas participen, sean protagonistas y líderes éticos en el desarrollo de la sociedad.

Otro componente que permite un constante refuerzo sobre la formación en ciudadanía son los medios de comunicación, entre ellos, las redes sociales, ya que este es un espacio llamativo, de gran influencia e interacción que utilizan los jóvenes y adolescentes para socializar; por lo tanto, y como lo manifiesta Terrones (2018), es importante reforzar en los jóvenes y adolescentes la construcción de un posicionamiento autónomo, relacionados con las lecturas realizadas en los medios de comunicación, buscando mantener una distancia crítica y reflexiva al momento de tomar decisiones o hacerse opiniones sobre los problemas sociales, políticos y culturales, con el fin de fortalecer la opinión propia.

\section{Conclusiones}

La investigación cumplió con el objetivo, ya que se pudo identificar que el personal docente de Educación Física contribuye a la formación en Competencias Fundamentales a través del desarrollo de las actividades lúdico deportivas, pero esta no se desarrolla de la forma como lo plantea el currículo dominicano, pues existen diferencias significativas de lo propuesto con lo desarrollado en la clase. Sin embargo, ante las dificultades presentadas, el profesorado está presto a solucionarlas de la mejor manera, en busca del bienestar del estudiantado, tomando como base su propia formación y creencias en valores sociales y culturales.

Es importante que el conjunto docente de Educación Física se vea a sí mismo como un instrumento clave en la mejora de la formación ciudadana, ante la comunidad y el entorno institucional (Martínez e Ibarrola, 2015); la educación debe permear las paredes de la escuela e involucrar actividades en la comunidad, en compañía de los líderes comunitarios, tutores, padres de familia y medios de comunicación (Peña, Herrera y Salazar, 2016).

Si bien, el currículo dominicano describe las Competencias Fundamentales, sería conveniente dotar al profesorado con instrumentos de medición, estrategias de evaluación y didáctica, para ser implementadas desde la clase de Educación Física.

Por parte de las entidades gubernamentales, deberían crear estrategias que permitan familiarizar al personal docente sobre la implementación de actividades específicas para el fortalecimiento las Competencias Fundamentales, al igual que la forma adecuada de hacer seguimiento para su desarrollo, esto permite unificar los criterios y así ser implementada en las instituciones educativas.

Los líderes y responsables del área administrativa de la Educación Física deberían prestar más apoyo y seguimiento, tanto a las escuelas como al profesorado de esta área, en la dotación oportuna de recursos e implementos, tanto deportivos como para el desarrollo de las actividades recreativas, necesarias para la formación en Competencias Fundamentales.

Se invita a desarrollar más estudios en el tema, con el fin de ampliar la mirada y conocer de primera mano las experiencias y resultados que se están elaborando en relación con las Competencias Fundamentales, para crear estrategias pedagógicas y experiencias reproducibles, tanto en el contexto escolar como fuera de este. 


\section{REFERENCIAS}

Agüero, S. (2015). El concepto de hacer trampa en el deporte organizado. Un inventario temático de problemas. FairPlay: Revista de filosofía, ética y derecho del deporte, (7), 155-181. Recuperado de: https://bit.ly/2UzktiK

Alarcón, J. y Donoso, S. (2018). ¿Nuevo paradigma en la política educativa chilena? Debates sobre educación y transformación social. Revista Educación, 42(2), 740-768. doi: https://doi.org/10.15517/REVEDU.V42I2.27 560

Asociación Médica Mundial [AMM]. (2019). Declaración de Helsinki de la AMM-Principios éticos para las investigaciones médicas en seres humanos. Recuperado de: https://bit.ly/2X $5 \mathrm{mSmS}$

Baggetta, P. y Alexander, P. A. (2016). Conceptualization and operationalization of executive function. Mind, Brain, and Education, 10(1), 10-33. doi: https://doi.org/10.1111/mbe.12100

Blanco, H. (2012). Análisis de datos cualitativos y atlas.ti: una experiencia de formación. Revista Universitaria: Docencia, Investigación e Innovación, 1(1), 103-114. Recuperado de: https://bit.ly/3bCRH6q

Bohórquez, M. y Checa, I. (2017). Diseño y validez de contenido de una entrevista para la evaluación psicológica de porteros de fútbol. Revista de Psicología Aplicada al Deporte y al Ejercicio Físico, 2(1), 1-12. doi: https://doi.or $\mathrm{g} / 10.5093 /$ rpadef2017a3

Cagigal, J. M. (1982). En torno a la educación por el movimiento. Revista Stadium, 96, 34-45. Recuperado de https ://bit.ly/2O $7 \mathrm{k} 3 \mathrm{fq}$

Cárcamo, H. (2015). Ciudadanía y formación ciudadana: ¿Qué ocurre en el aula donde se forma el futuro profesorado de educación primaria de una universidad pública de Madrid?. Revista Electrónica Educare, 19(3), 31-44. doi: h ttps://doi.org/10.15359/ree.19-3.3

Delgado, M. y Castrillo, L. (2015). Efectividad del aprendizaje cooperativo en contabilidad: una contrastación empírica. Revista de Contabilidad, 18(2), 138-147. Recuperado de: https://doi.org/10.1016/j.rcsar.2014.05.003

Folgueiras, P. y Ramírez, C. (2017). Elaboración de técnicas de recogida de información en diseños mixtos. Un ejemplo de estudio en aprendizaje-servicio. Revista d'Innovació i Recerca en Educació, 10(2), 64-78. doi: https://doi.org /10.1344/reire2017.10.218069

Guerrero, M. (2016). La Investigación Cualitativa. INNOVA Research Journal, 1(2), 1-9. doi: https://doi.org/10.33 890/innova.v1.n2.2016.7

Herrera, G. (2016). La formación para la investigación de los estudiantes de Medicina: la brecha entre el currículo formal y el currículo oculto. Revista de Ciencias Médicas de Pinar del Río, 20(3), 134-143. Recuperado de http s://bit.ly/3aDogkH

Lamoneda, J. y Huertas, F. (2017). Efectos de un programa de juego limpio aplicado a jóvenes futbolistas para la mejora de la deportividad. Revista Iberoamericana de Psicología del Ejercicio y el Deporte, 12(1), 107-119. Recuperado de https://bit.ly/3bD6w9c

Levoratti, A. (Diciembre de 2014). El juego y el deporte formando ciudadanos: Un análisis sobre los contenidos de la Educación Física en la Educación Secundaria de la provincia de Buenos Aires 2006-2014. En M. Busso (Presidencia), VIII Jornadas de Sociología de la UNLP. Llevado a cabo en Departamento de Sociología de la Facultad de Humanidades y Ciencias de la Educación de la Universidad Nacional de la Plata llevado, Ensenada, Argentina. Recuperado de https://bit.ly/39C4dSf

López, V. M., Pérez, D., Manrique, J. C. y Monjas, R. (2016). Los retos de la Educación Física en el Siglo XXI. Retos. Nuevas tendencias en Educación Física, Deporte y Recreación, (29), 182-187. Recuperado de https://bit.ly/2JwZ TZQ

Maldonado, M.E. (2018). El aula, espacio propicio para el fortalecimiento de competencias ciudadanas y tecnológicas. Sophia, 14(1), 39-50. doi: https://doi.org/10.18634/sophiaj.14v.1i.822

Marín, A., Hernández, E. y Flores, J. (2016). Metodología para el análisis de datos cualitativos en investigaciones orientadas al aprovechamiento de fuentes renovables de energía. Revista Arbitrada Interdisciplinaria Koinonía, I(1), 60-75. Recuperado de https://bit.ly/2W6LN7G 
Martínez A. e Ibarrola S. (2015). Liderazgo del profesor: objetivo básico de la gestión educativa. Revista Iberoamericana De Educación, 67, 55-70. doi: https://doi.org/10.35362/rie670205

Ministerio de Educación de la República Dominicana [MINERD]. (2016), Diseño Curricular Nivel Primario, Modalidad Académica, Segundo Ciclo 4to, 5to y 6to, Versión Preliminar Para Revisión y retroalimentación. Recuperado de https://bit.ly/3ffoTD7

Monzonís, N. y Capllonch, M. (2015), Mejorar la competencia social y ciudadana: innovación desde Educación Física y tutoría. RETOS. Nuevas Tendencias en Educación Física, Deporte y Recreación, (28), 256-262. Recuperado de: https://bit.ly/2RiBP1p

Mossop, L., Dennick, R., Hammond, R. y Robbé, I. (2013). Analysing the hidden curriculum: use of a cultural web. Medical education, 47(2), 134-143. doi: https://doi.org/10.1111/medu.12072

Ospina, V. (2016). El docente del nivel inicial: retos para la formación profesional y continúa. Educación y Humanismo, 18(30), 107-122. doi: https://doi.org/10.17081/eduhum.18.30.1325

Otzen, T. y Manterola, C. (2017). Técnicas de Muestreo sobre una Población a Estudio. International Journal of Morphology, 35(1), 227-232. doi: https://doi.org/10.4067/S0717-95022017000100037

Pacheco, Y.B. (2017). Estar, ser y con-vivir en la escuela. La violencia escolar desde las voces de estudiantes y docentes. (Tesis Doctoral Inédita). Universidad de Sevilla, Sevilla. Recuperado de https://bit.ly/3bXNElo

Palomares, J., Dharmadi, M. A., Sulistia, N., Collado, D. y Padial, R. (2018). La acción docente en la protección sociocomunitaria de los juegos populares de Buleleng-Bali. Revista Brasileira de Ciências do Esporte, 40(2), 177-183. doi: https://doi.org/10.1016/j.rbce.2018.01.018

Peña, C., Herrera, L. y Salazar, J. (2016). Participación ciudadana a través de los medios de comunicación como legitimación de la democracia participativa en Colombia. Advocatus, (26), 145-153. doi: https://doi.org/10.18 041/0124-0102/advocatus.26.942

Pherez, G., Vargas, S. y Jerez, J. (2018). Neuroaprendizaje, una propuesta educativa: herramientas para mejorar la praxis del docente. Civilizar Ciencias Sociales y Humanas, 18(34), 149-166. Recuperado de https://bit.ly/2ZRI2oh

Prada, M. J. (2018). Descripción de las competencias ciudadanas que presentan los jóvenes y adolescentes. Revista Caribeña de Investigación Educativa (RECIE), 2(1), 51-66. doi: https://doi.org/10.32541/recie.2018.v2i1.pp5 $1-66$

Schulz, W., Ainley, J., Fraillon, J., Kerr, D. y Losito, B. (2010). Resultados iniciales del Estudio Internacional de Educación Civica y Ciudadana de la IEA. Amsterdam: Asociación Internacional para la Evaluación del Logro Educativo. Recuperado de: https://bit.ly/2yKnUun

Terrones, A. L. (2018). Pensamiento dominante, educación y medios de comunicación. Sophia, Colección de Filosofía de la Educación, (24), 313-336. doi: https://doi.org/10.17163/soph.n24.2018.10

Zambrano, E. L. (2018). Prácticas pedagógicas para el desarrollo de competencias ciudadanas. Revista electrónica de investigación educativa, 20(1), 69-82. doi: https://doi.org/10.24320/redie.2018.20.1.1409

\section{BY-NC-ND}

